\title{
Change in Entropy of Spinning Black Holes Due to Corresponding Change in Mass in XRBs
}

\author{
Dipo Mahto1, Anuradha Kumari² \\ ${ }^{1}$ Department of Physics, Marwari College, T. M. B. University, Bhagalpur, India \\ ${ }^{2}$ Department of Physics, T. M. B. University, Bhagalpur, India \\ Email: dipomahto@hotmail.com, anuphy1987@gmail.com
}

How to cite this paper: Mahto, D. and Kumari, A. (2018) Change in Entropy of Spinning Black Holes Due to Corresponding Change in Mass in XRBs. International Journal of Astronomy and Astrophysics, 8, 171-177.

https://doi.org/10.4236/ijaa.2018.82012

Received: January 8, 2018

Accepted: June 6, 2018

Published: June 11, 2018

Copyright $\odot 2018$ by authors and Scientific Research Publishing Inc. This work is licensed under the Creative Commons Attribution International License (CC BY 4.0).

http://creativecommons.org/licenses/by/4.0/

\begin{abstract}
The present paper gives a theoretical model for the change in entropy of spinning black holes due to change in mass to use the first law of black hole mechanics for unit spinning parameter and angular momentum in XRBs. This shows that the entropy change with respect to the mass of uncharged spinning black holes is essentially the function of mass and their surface gravity are lesser than to that of the non-spinning black holes.
\end{abstract}

\section{Keywords}

Entropy Change, Spinning Parameters and Angular Momentum

\section{Introduction}

Classically, the black holes are perfect absorbers and do not emit anything; their temperature is absolute zero. However, in quantum theory black holes emit Hawking radiation with a perfect thermal spectrum [1]. The laws of black hole Physics describe the behaviour of a black hole in close analogy to the laws thermodynamics by relating mass to energy, area to entropy, and surface gravity to temperature [2]. The general formula for the entropy due to Bekenstein and Hawking provides a deep connection between quantum mechanics, general relativity and thermodynamics [3]. In 2006, Zhao Ren et al. provided a general method for the discussion on quantum corrections to Bekenstein and Hawking Entropy [4]. In 2011, Mahto et al. proposed a model for the change in energy and entropy of Non-spinning black holes to use the first law of black hole mechanics and mass-energy equivalence relation [5]. In 2013, Mahto et al. proposed also a model for the change in entropy of Non-spinning black holes with respect to the radius of event horizon in XRBs by using the first law of the black hole mechan- 
ics with the relation for the entropy change $\mathrm{d} S=8 \pi \mathrm{d} M \quad$ [6]. In 2015, Mahto et al. gave a model for classical statistical entropy of black hole using M-B Statistics and showed that the classical statistical entropy of black hole is directly proportional to the area of event horizon [7].

In the present paper, we have proposed a model for the change in entropy of spinning black holes due to change in mass to use the first law of black hole mechanics for unit spinning parameter and angular momentum and also calculated their values for different test spinning black holes in XRBs.

\section{Theoretical Discussion}

The first law of black hole mechanics is simply an identity relating the change in mass $M$, angular momentum $J$, horizon area $A$ and charge $Q$, of a black hole. The first order variations of these quantities in the vacuum satisfy [8].

$$
\delta M=\frac{k}{8 \pi} \delta A+\Omega \delta J-v \delta Q .
$$

where $\Omega=$ Angular velocity of the horizon. $v=$ difference in the electrostatic potential between infinity and horizon.

In this more general context, the first law of black hole mechanics is seen to be a direct consequence of an identity holding for the variation of the Noether current. The general form of the first law takes the form [1]

$$
\begin{gathered}
\delta M=\frac{k}{2 \pi} \delta S+\Omega \delta J \\
\text { or } \delta S=\frac{2 \pi}{\kappa}[\delta M-\Omega \delta J]
\end{gathered}
$$

The above equation shows a model for change in entropy due to corresponding change in mass and angular momentum of black holes and depends on the surface gravity.

\section{Surface Gravity}

The surface gravity as an entity playing a role in black hole physics is analogous to temperature in thermodynamics and can be defined for stationary asymmetric black holes in asymptotically flat space time. The surface gravity for the Kerr-Newman solution is [8]

$$
\kappa=\frac{\sqrt{M^{2}-Q^{2}-J^{2} / M^{2}}}{2 M^{2}-Q^{2}+2 M \sqrt{M^{2}-Q^{2}-J^{2} / M^{2}}} .
$$

where $Q$ is the electric charge, Jis the angular momentum.

For uncharged black holes

$$
Q=0
$$

Using the above equation into Equation (4), we have

$$
\kappa=\frac{\sqrt{M^{2}-J^{2} / M^{2}}}{2 M^{2}+2 M \sqrt{M^{2}-J^{2} / M^{2}}} .
$$


Solving, we have

$$
\begin{gathered}
\kappa=\frac{\sqrt{M^{4}-J^{2}}}{2 M^{3}+2 M \sqrt{M^{4}-J^{2}}} . \\
\text { Now } \sqrt{M^{4}-J^{2}}=\left(M^{4}-J^{2}\right)^{1 / 2}=M^{2}\left(1-\frac{J^{2}}{M^{4}}\right)^{1 / 2}
\end{gathered}
$$

The square root expression then expanded by use of the binomial theorem:

$$
=M^{2}\left(1-\frac{1}{2} \frac{J^{2}}{M^{4}}+\frac{1}{8}\left(\frac{J^{2}}{M^{4}}\right)^{2}-\frac{1}{16}\left(\frac{J^{2}}{M^{4}}\right)^{3}+\cdots\right)
$$

Neglecting the higher power terms, we have

$$
\begin{aligned}
& \text { Now } \sqrt{M^{4}-J^{2}}=M^{2}\left(1-\frac{J^{2}}{2 M^{4}}\right) \\
& \text { Also } 2 M^{3}+2 M \sqrt{M^{4}-J^{2}}=2 M^{3}+2 M^{3}\left(1-\frac{J^{2}}{M^{4}}\right) \\
& \text { Or } 2 M^{3}+2 M \sqrt{M^{4}-J^{2}}=4 M^{3}\left(1-\frac{J^{2}}{4 M^{4}}\right)
\end{aligned}
$$

Putting (10) and (11) in Equation (7), we have

$$
\begin{gathered}
\kappa=\frac{M^{2}\left(1-\frac{J^{2}}{2 M^{4}}\right)}{4 M^{3}\left(1-\frac{J^{2}}{4 M^{4}}\right)}=\frac{\left(1-\frac{J^{2}}{2 M^{4}}\right)}{4 M\left(1-\frac{J^{2}}{4 M^{4}}\right)} \\
\text { or } \kappa=\frac{\left(1-\frac{J^{2}}{2 M^{4}}\right)\left(1-\frac{J^{2}}{4 M^{4}}\right)^{-1}}{4 M}=\frac{\left(1-\frac{J^{2}}{2 M^{4}}\right)\left(1+\frac{J^{2}}{4 M^{4}}\right)}{4 M}
\end{gathered}
$$

The solution of above equation gives the following result.

$$
\kappa=\frac{1}{4 M}\left[1-\frac{J^{2}}{4 M^{4}}+\frac{J^{4}}{8 M^{8}}\right]
$$

For massive/super massive black holes, the term $\mu^{4} / 8 M^{4}$ can be neglected.

$$
\kappa=\frac{1}{4 M}\left[1-\frac{J^{2}}{4 M^{4}}\right] \text {. }
$$

For a black holes of zero angular momentum, $J=0$

$$
\kappa=\frac{1}{4 M}
$$

The above equation shows the surface gravity of black hole in Schwarzschild case [9].

From Equation (15), we have

$$
\frac{1}{\kappa}=4 M\left[1+\frac{J^{2}}{2 M^{4}}\right]
$$


The angular momentum of black hole is defined by the following equation [10]

$$
J=a_{*} G M^{2} / c
$$

Throughout this research paper, assuming $G=c=1$ [9] [10], we have

$$
J=a_{*} M^{2} .
$$

The radius is smaller in the case of spinning black holes, tending to $G M / c^{2}$ as $a^{*}=1[9]$ and hence, we have

$$
J=M^{2}
$$

Using the above equation into Equation (17), we have

$$
\begin{aligned}
\frac{1}{\kappa} & =6 M \\
\text { Or } \kappa & =\frac{1}{6 M}
\end{aligned}
$$

The Equation (16) shows the surface gravity of non-spinning black holes/Schwarzschild black holes, while Equation (22) shows the surface gravity of spinning black holes with spin parameter $a^{*}=1$. This means that the surface gravity of spinning $\mathrm{BH}$ with $a^{*}=1$ is lesser than to that of non-spinning black holes of the same mass.

Using the above Equation (22) into Equation (3), we have

$$
\delta S=12 \pi M[\delta M-\Omega \delta J] .
$$

For unit angular velocity, the above equation takes place

$$
\delta S=12 \pi M[\delta M-\delta J] .
$$

Differentiating equation (20), we have

$$
\delta J=2 M \delta M .
$$

Using the above equation into Equation (24), we have

$$
\begin{gathered}
\delta S=12 \pi M[\delta M-2 M \delta M] \\
\delta S=12 \pi M[1-2 M] \delta M \\
\frac{\delta S}{\delta M}=12 \pi M[1-2 M]
\end{gathered}
$$

The term 1 in compared with the mass of black holes is negligible and can be neglected.

$$
\begin{gathered}
\frac{\delta S}{\delta M}=-24 \pi M^{2} . \\
\left|\frac{\delta S}{\delta M}\right|=24 \pi M^{2} \\
\left|\frac{\delta S}{\delta M}\right| \alpha M^{2} .
\end{gathered}
$$




\section{Data in Support for the Mass of Black Holes}

There are two categories of black holes classified on the basis of their masses clearly very distinct from each other, with very different masses $M \sim 5-20 M_{\odot}$ for stellar-mass black holes in X-ray binaries [10].

On the basis of the data mentioned above regarding the mass of sun and black holes in XRBs, we have calculated change in entropy of spinning black holes due to corresponding change in mass for different test spinning black holes.

\section{Result and Discussion}

In the present work, firstly we have started our work with the general form of first law of black hole mechanics to give a model for change in entropy of uncharged spinning black holes by the relation $\delta S=\frac{2 \pi}{\kappa}[\delta M-\Omega \delta J]$.

Secondly, we have calculated the surface gravity of uncharged spinning black holes by putting $\mathrm{d} Q=0$ in the Kerr solution and obtained its value by this relation $\kappa=\frac{1}{6 M}$.

Thirdly, we have used a relation between the angular momentum $(J)$ and mass of spinning black holes to convert the change in the angular momentum in terms of change in mass of the spinning black holes for unit spin parameter and angular velocity.

The work is further extended with proper mathematical operation to obtain the change in entropy of uncharged spinning black hole with respect to the change in mass $(\mathrm{d} S / \mathrm{d} M)$ in terms of mass by the equation $\left|\frac{\delta S}{\delta M}\right|=24 \pi M^{2}$.

The final Equation (30) shows that the magnitude of the change in entropy due to change in mass of spinning black holes in XRBs is directly proportional to the square of the mass of the spinning black holes.

Fourthly, we have plotted the graph with the help of Table 1, between the mass of spinning black holes and corresponding the magnitude of the change in entropy with change in mass of spinning black holes in XRBs as shown in the Figure 1 and shows that the magnitude of change in entropy with change in mass increases with the increasing the mass of different test spinning black holes.

\section{Conclusions}

We have concluded the following facts during the research work:

1) The surface gravity of uncharged spinning black holes is lesser than to that of the non-spinning black holes.

2) The magnitude of the change in entropy with change in mass of spinning black holes is directly proportional to the square of their masses.

3) The magnitude of the change in entropy with change in mass of uncharged spinning black holes is essentially the function of their masses. 
Table 1. Change in mass of spinning black holes due to corresponding change in angular momentum in XRBs for $a^{*}=1$ and $(\Omega=1)$.

\begin{tabular}{cccc}
\hline Sl. No & $\begin{array}{c}\text { Mass of black holes }(M) \text { in } \\
\text { terms of solar mass }\left(M_{\odot}\right)\end{array}$ & $\begin{array}{c}\text { Spin parameter } \\
\left(a^{*}=1\right) \& \text { angular } \\
\text { velocity }(\Omega=1)\end{array}$ & $\begin{array}{c}\mathrm{d} S / \mathrm{d} M \\
\text { (Joule per Kelvin per solar mass 2) }\end{array}$ \\
\hline 1 & $5 M_{\odot}$ & 1 & $7.536 \times 10^{3}$ \\
2 & $6 M_{\odot}$ & 1 & $10.851 \times 10^{3}$ \\
3 & $7 M_{\odot}$ & 1 & $14.770 \times 10^{3}$ \\
4 & $8 M_{\odot}$ & 1 & $19.292 \times 10^{3}$ \\
5 & $9 M_{\odot}$ & 1 & $24.416 \times 10^{3}$ \\
6 & $10 M_{\odot}$ & 1 & $30.144 \times 10^{3}$ \\
7 & $11 M_{\odot}$ & 1 & $3.474 \times 10^{3}$ \\
8 & $12 M_{\odot}$ & 1 & $43.407 \times 10^{3}$ \\
9 & $13 M_{\odot}$ & 1 & $50.943 \times 10^{3}$ \\
10 & $14 M_{\odot}$ & 1 & $59.082 \times 10^{3}$ \\
11 & $15 M_{\odot}$ & 1 & $67.824 \times 10^{3}$ \\
12 & $16 M_{\odot}$ & 1 & $77.168 \times 10^{3}$ \\
13 & $17 M_{\odot}$ & 1 & $87.116 \times 10^{3}$ \\
14 & $18 M_{\odot}$ & 1 & $97.666 \times 10^{3}$ \\
15 & $19 M_{\odot}$ & 1 & $108.819 \times 10^{3}$ \\
\hline & $20 M_{\odot}$ & $120.576 \times 10^{3}$ \\
\hline
\end{tabular}

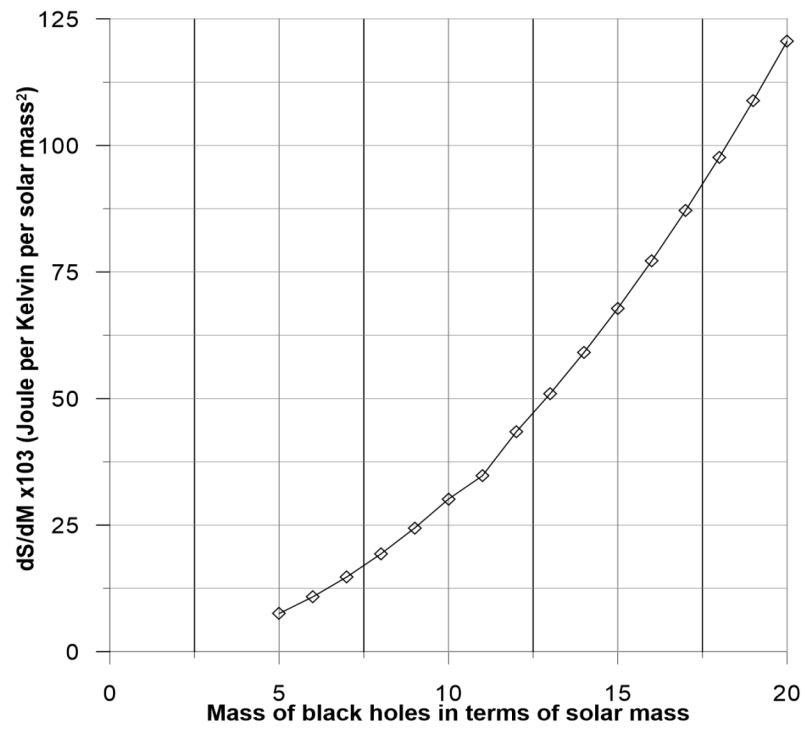

Figure 1. The graph plotted between the mass of black holes in terms of solar mass and their corresponding change in entropy relative to the change in mass for XRBs.

4) On increasing the mass of spinning black holes, their corresponding change in magnitude of the entropy with change in mass increases in the case of unit 
spin parameter and angular velocity.

\section{Acknowledgements}

The authors are very grateful to reviewers to find out the errors in the original manuscript and providing the constructive suggestions. This paper is also devoted in memory of great scientist Stephen Hawking (March 14, 2018).

\section{References}

[1] Wald, R.M. (2001) The Thermodynamics of Black Holes. Living Rev. in Relativity. https://www.springer.com/cn/livingreviews

[2] Hawking, S.W. (1975) Particle Creation by Black Hole. Communications in Mathematical Physics, 43, 199-220. https://doi.org/10.1007/BF02345020

[3] Dabholkar, A. (2006) Black Hole Entropy in String Theory: A Window into the Quantum Structure of Gravity. In: The Legacy of Albert Einstein, Current Science, 47-68. https://doi.org/10.1142/9789812772718_0004

[4] Ren, Z., Hai-Xia, Z. and Shuang-Qi, H. (2006) General Logarithmic Corrections to Bekenstein and Hawking Entropy. arxiv-gr-qc0609080.

[5] Mahto, D., Kumari, K., Sah, R.K. and Singh, K.M. (2011) Study of Non-Spinning Black Holes with Reference to the Change in Energy and Entropy. Astrophysics and Space Science, 337, 685-691. https://doi.org/10.1007/s10509-011-0883-7

[6] Mahto, D., Prakash, V., Prasad, U. And Singh, B.K. (2013) Change in Entropy of Non-Spinning Black Holes w.r.t. the Radius of Event Horizon in XRBs. Astrophysics and Space Science, 343, 153-159. https://doi.org/10.1007/s10509-012-1219-y

[7] Mahto, D., Prakash, V., Singh, K.M. and Kumar, B. (2015) Classical Statistical Entropy of Black Hole. American Journal of Theoretical and Applied Statistics, 4, 1-18. https://doi.org/10.11648/j.ajtas.s.2015040101.13

[8] Bardeen, J.M., Carter, B. and Hawking, S.W. (1973) The Four Laws of Black Hole Mechanics. Communications in Mathematical Physics, 31, 161-170. https://doi.org/10.1007/BF01645742

[9] Transchen, J. (2000) An Introduction to Black Hole Evaporation. arXiv: gr-qc/0010055V.

[10] Narayan, R. (2005) Black Holes in Astrophysics. New Journal Physics, 7, 1-31. https://doi.org/10.1088/1367-2630/7/1/199 